\title{
Management of fecal incontinence - focus on a vaginal insert for bowel control
}

This article was published in the following Dove Press journal:

Medical Devices: Evidence and Research

10 May 2016

Number of times this article has been viewed

\author{
Eric R Sokol \\ Department of Obstetrics and \\ Gynecology, Stanford University \\ School of Medicine, Stanford, CA, USA
}

Correspondence: Eric R Sokol Department of Obstetrics and Gynecology, Stanford University School of Medicine, 300 Pasteur Drive, Room G332, Stanford, CA 94305, USA

Tel + I 6507364137

Fax + I 6507237737

Email esokol@stanford.edu
Abstract: Fecal incontinence, also referred to as accidental bowel leakage, is a debilitating condition that impacts quality of life in a significant number of women. Current treatments for fecal incontinence include behavioral modification, biofeedback, drug therapy, and invasive surgical procedures. However, these treatments have suboptimal efficacy due to patient adherence, variability of presentation across patients, cost, and additional health risks. A vaginal bowel control system (Eclipse ${ }^{\mathrm{TM}}$ System) was developed to offer a low-risk, effective, and patient-managed approach to treating accidental bowel leakage. The vaginal bowel control system consists of a vaginal insert and user-controlled, pressure-regulated pump. Once inflated, the balloon of the vaginal insert is directed posteriorly to occlude the rectum, allowing the woman to immediately regain control of bowel function. This article will introduce the design evolution and feasibility studies of the Eclipse System. In addition, this review will discuss the results from a recent clinical trial that demonstrated the safety and efficacy of the vaginal bowel control system in managing fecal incontinence and other symptoms of bowel dysfunction.

Keywords: accidental bowel leakage, fecal urgency, vaginal bowel control system, Eclipse System, bowel function

\section{Overview of accidental bowel leakage}

Accidental bowel leakage (ABL), a patient-centered term for fecal incontinence (FI), ${ }^{1}$ is a socially debilitating condition that is characterized by involuntary loss of liquid or solid stool. In addition, ABL severity is influenced by frequency of leakage, stool consistency, and urgency. ABL prevalence rates range from $7 \%$ to $15 \%$ of communitydwelling women in the Western world with an estimated 20 million US women living with the condition. ${ }^{2}$ In addition to increased rates with aging, women who have experienced pregnancy, childbirth, irritable bowel syndrome, or nerve damage to the pelvic region have greater risk for developing bowel dysfunction. ${ }^{3-5}$ Given that these rates may be underreported given some women's resistance to seeking consultation, ABL represents a large unmet medical need in women's health.

The pathophysiology of ABL is multifactorial and may vary by patient. Normal bowel control relies on anatomical integrity of internal anal sphincter, external anal sphincter, and puborectalis muscles; intact rectoanal sensation; and sufficient muscle response (contraction/relaxation), including the ability of the rectum to store fecal load until voluntary release (rectal compliance) ${ }^{2}$ Dysfunction in any of these components independently or in combination can contribute to development of $\mathrm{ABL}$, with factors ranging from iatrogenic, neurogenic (eg, peripheral

submit your manuscript | www.dovepress.com 
neuropathy, central nervous system disease), or comorbidities (eg, anorectal inflammation associated with Crohn's disease, ulcerative colitis). In women, ABL is often due to damaged or weakened anal sphincters, which can result from childbirth, reduced pelvic floor function, disturbed rectal sensation, or decreased rectal capacity. ${ }^{1,6,7}$ Furthermore, aging is associated with weakness of internal and/ or external sphincters. ${ }^{8}$

ABL significantly impacts daily life and is associated with decreased self-confidence, self-respect, modesty, and composure. Patient responses to quality-of-life (QOL) questionnaires indicate that $\mathrm{ABL}$ restricts social life due to anxiety associated with knowing toilet location, hygiene/odor issues, coping strategies, physical activities, and embarrassment. ${ }^{6,9,10}$ A woman with ABL may withdraw from social situations due to social stigma and discomfort in sharing her condition with family and friends. Barriers to seeking care include lack of understanding, embarrassment, belief that $\mathrm{ABL}$ is a normal part of aging, lack of appropriateness of care providers, and doubt that treatments can help. ${ }^{11} \mathrm{QOL}$ scores are highly correlated to ABL severity, emphasizing the need for effective treatments to manage bowel function to continue daily activities.

Most women with ABL do not seek medical treatment, utilizing self-managing strategies such as absorbent pads and diet restriction. Upon clinical counsel, conservative treatment options for ABL include dietary changes, medications, and pelvic floor muscle training with or without biofeedback. However, less than $25 \%$ of patients describe adequate relief with conservative management intervention. ${ }^{12,13}$ In patients who have failed conservative therapy, invasive surgical options include sphincteroplasty, sacral nerve stimulation, injection of bulking agents, radiofrequency energy delivery, and placement of an artificial bowel sphincter. The correction of rectal prolapse, including rectopexy, is an additional possible surgical treatment modality for FI. Surgical interventions for FI can be costly and invasive, can require complex management or the need for additional follow-up, and are associated with a $30 \%-50 \%$ failure rate during long-term evaluation. ${ }^{14-19}$

\section{Design and features of the Eclipse System, a vaginal bowel control system for $A B L$}

Given limitations in existing therapies, an unmet medical need remains for new, effective, and minimally invasive treatments for ABL. A vaginal bowel control (VBC) system (Eclipse ${ }^{\mathrm{TM}}$ System; Pelvalon, Sunnyvale, CA, USA) was developed to provide a low-risk and patient-managed approach to treating ABL, which could provide immediate effectiveness. Specifically, the Eclipse System consists of a vaginal insert and user-controlled, pressure-regulated pump (Figure 1). The vaginal insert consists of a siliconecoated stainless steel base and posterior-directed, dual-layer balloon that provides reversible occlusion of the rectum, enabling the user to regain control of her bowel function (Figure 2).

The insert, which is available in a range of base and balloon sizes, does not need to be removed for defecation, but can simply be deflated. The device can be fit and inserted in the clinic, then is easily controlled and removed or reinserted by the patient. For insertion, the user folds the insert along its length and places it into the vagina, balloon-end first and directed posteriorly (Figure 3). It is recommended to remove the insert for cleaning once per week, as well as prior to sexual intercourse. For women who choose to use the insert during menstruation, daily cleaning is recommended. The tube, which extends outside of the vagina to allow for inflation and deflation, can be lengthened or shortened to patient preference, using a connector on the tube. The Eclipse System was initially approved by the US Food and Drug Administration in February 2015. This therapy fills a gap in the continuum of treatment for FI between conservative medical management and invasive surgery (Figure 4).

Medical device development typically involves multiple stages of evolution and evaluation driven by investigational trial use and standard care delivery. Following bench top and cadaveric testing for initial concept and design, optimization of clinical delivery and efficacy of the VBC system was achieved through staged human subject evaluations during short-term in-clinic use and short-term at-home wear, as described in the following section.

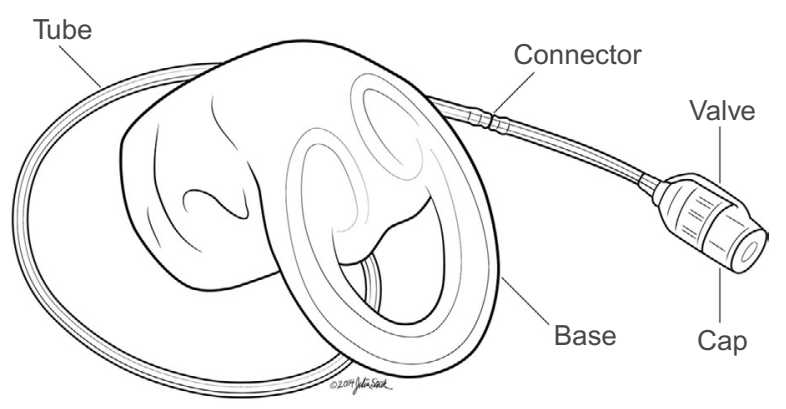

Figure I The vaginal bowel control (VBC) insert.

Note: The VBC insert consists of a silicone-coated stainless steel base and posterior-directed, dual-layer balloon that connects to a user-controlled, pressureregulated pump. Printed with permission from @ Julia Stack. 

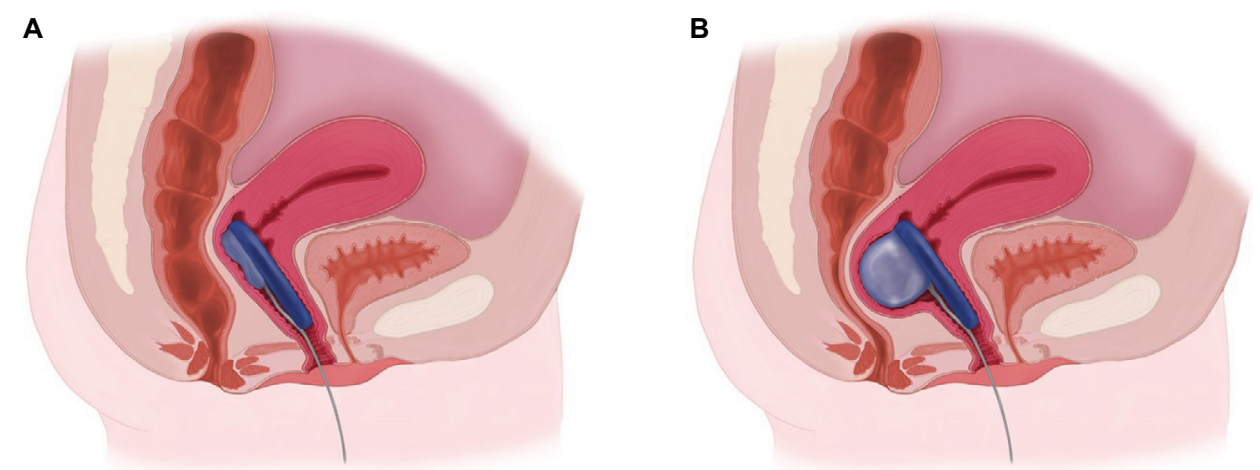

Figure 2 The vaginal bowel control insert deflated $(\mathbf{A})$ and inflated $(\mathbf{B})$.

Notes: Upon inflation, the dual-layer balloon provides reversible occlusion of the rectum, enabling the user to regain control of her bowel function. The insert, which is available in a range of base and balloon sizes, does not need to be removed for defecation, but can simply be deflated (courtesy of Pelvalon).
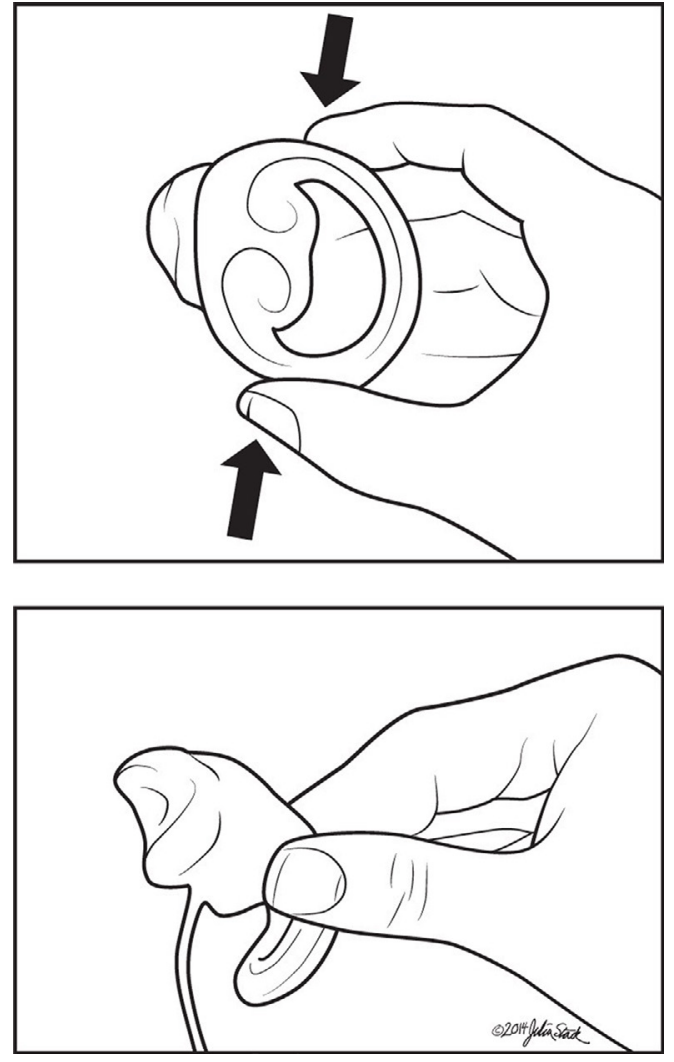

Figure 3 Folding the vaginal bowel control insert for placement. Note: Printed with permission from $@$ Julia Stack.

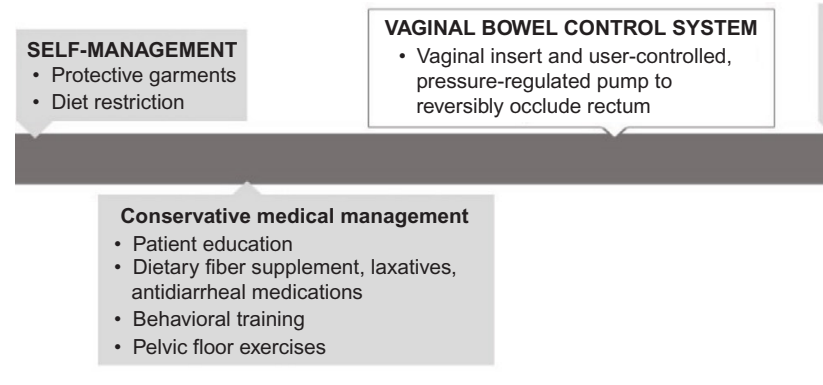

BIOFEEDBACK

BULKING AGENT INJECTION

provide awareness of physiologica responses

\section{Clinical data and design evolution of the Eclipse System Initial feasibility studies}

We conducted the first-in-human study as a single-center prospective, open-label clinical study that included 13 women who sought treatment for ABL, defined as at least one stool leakage (solid or liquid) episode per month. ${ }^{20,21}$ Once consented, subjects were seen for a single clinic visit during which demographics, medical history, and questions regarding subjects' FI symptoms were collected in a baseline questionnaire. A standard pelvic examination (vaginal speculum and digital rectal) was performed to assess the subject's pelvic health prior to the initial fitting. During the fitting, the physician vaginally inserted the VBC device, assessing the fit and position of the insert and degree of rectal occlusion. Finally, the subject was asked to assess comfort in both the inflated and deflated state and after various types of subject movement (eg, sitting, standing, and ambulating). In addition, subjects were fitted with multiple sizes of inserts during their study visit to determine the most appropriate configuration, and comfort was assessed at various inflation pressures.

Physician examination confirmed that the inflated state of the VBC insert provided majority occlusion of the rectum.

Figure 4 Treatment options for accidental bowel leakage. Note: Data from Whitehead et al. ${ }^{20}$ 
Overall, more than half of patients reported "no discomfort" during both deflated and inflated states of the vaginal insert. Furthermore, the majority of the patients indicated that they would wear the insert if allowed to continue participation beyond this single-visit study. No adverse events were reported. This first-in-woman study provided evidence that the novel concept of a VBC system for ABL was comfortable, well tolerated by subjects with ABL, and showed objective evidence of occluding the rectum.

A feasibility study consisting of staged, clinical evaluations was conducted to evaluate extended patient use of the VBC system in an outpatient setting and to confirm appropriateness of clinical protocol changes initiated following the first-in-woman study. Women with self-reported ABL extended the VBC system wear period to 1 week up to 1 month. A majority of women indicated desire for continued use after completion of the study, if allowed. Of the few adverse events, all were considered minor and resolved. (unpublished data, Takase-Sanchez M, 2014).

During the course of the first-in-human and feasibility studies, different versions of the device were evaluated, leading to enhanced comfort, stability, and effectiveness. Additionally, the cosmetic appearance of the system was modified to improve its appeal to patients. These different device versions integrated new designs in base shape and size, balloon shape and size, and the relative positioning of base and balloon. The softness and shape of the silicone covering was also designed to improve patient comfort, and the flexibility of the base was improved for ease of insertion and removal. Finally, a new pump (introduced commercially) was designed to provide improved ease of use, elegance, and discretion for women using the therapy (Figure 5).

The feasibility studies also examined the various questionnaires, scores, and assessments available to evaluate ABL severity and symptoms. For future trials, it was determined that a 2-week bowel diary provided the appropriate level of information about device effectiveness for both accident reduction and change in important QOL-impacting symptoms such as fecal urgency, frequency, stool consistency, and completeness of evacuation. Additionally, validated and condition-specific QOL scores were identified as meaningful to the patient experience with the device: the Fecal Incontinence Quality of Life, Modified Manchester Health Questionnaire, as well as the Patient Global Impression of Improvement.

\section{The LIFE clinical trial: safety and efficacy}

A multicenter, prospective clinical trial was conducted to determine therapeutic efficacy and safety (primary end

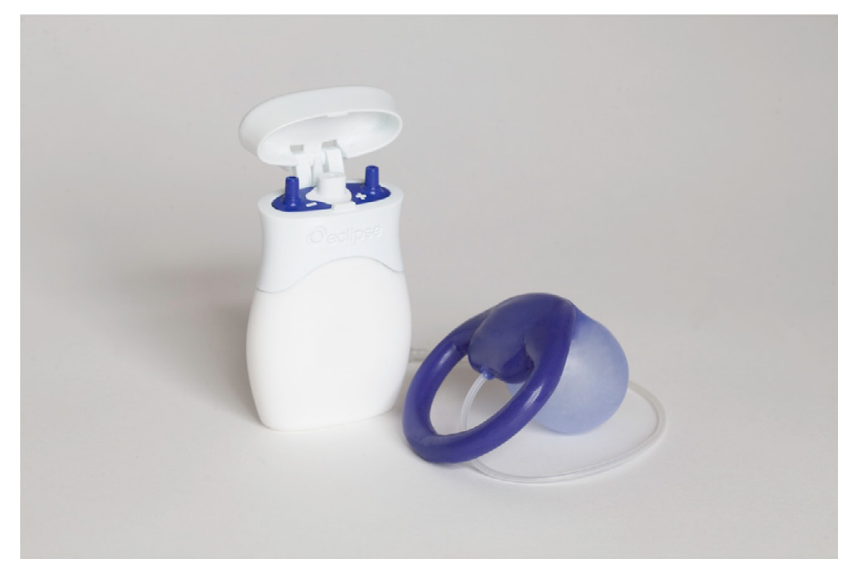

Figure 5 Updated vaginal bowel control system that enhances fit, stability, comfort, and appearance based on the feasibility studies (courtesy of Pelvalon).

points) of the VBC system for women with ABL (LIFE Study, ClinicalTrials.gov, http://www.clinicaltrials.gov, NCT01655498). ${ }^{22}$ Participant eligibility was determined using 2-week bowel diaries (baseline) that recorded the severity, consistency, and associated urgency of incontinent episodes. During the in-clinic fitting process, clinicians attempted to find a size and shape of the vaginal insert that was comfortable and could be appropriately positioned in the vagina, selecting from round or elliptical shapes in a range of sizes. In addition, the size of the inflatable balloon was determined based on vaginal caliber and rectal examination. If the insert was determined to be stable in both deflated and inflated position during the in-clinic visit, the subject went home for a 1-week trial. If the insert was comfortable, then the subject entered the clinical trial. However, if the insert was uncomfortable, the patient could return to the clinic for a refitting and another trial week. Patients exited the study after four failed trials.

Eligible participants with successful insert fittings completed at least 1 month of VBC system use and documented ABL symptoms during the final 2 weeks of the wear period (treatment bowel diary). Therapeutic efficacy was determined by comparing the frequency of FI episodes between the baseline and bowel diaries with treatment success defined as greater than $50 \%$ reduction in incontinent episodes. Adverse events were evaluated to assess safety. In addition, QOL measurements were collected as secondary outcomes. Women who completed the 1-month period were invited to extend use for a 2-month extended period (3 months total use).

Richter et al reported the primary outcomes from the trial, demonstrating successful efficacy and safety of the $\mathrm{VBC}$ system for treatment of ABL in women. ${ }^{23}$ Of the 110 
women who consented to participate and advanced to the fitting phase, 61 women entered the treatment period with $93 \%$ of subjects $(n=56)$ completing the 1 -month period per protocol (three withdrew from the trial, two did not fully complete treatment diaries). Eighty-six percent of the per protocol population had treatment success $(\geq 50 \%$ reduction in episodes) with a significant decrease in ABL frequency from 11.66 \pm 9.65 per 2 weeks (baseline) to 2.16 \pm 2.9 episodes per 2 weeks with VBC system use for 1 month. Forty-one percent achieved complete continence. Forty-four women continued VBC system use for a total of 3 months. The majority of these participants $(86 \%)$ met treatment success, with 20 women (46\%) reporting complete continence (Figure 6).

Mild and moderate adverse events experienced during the fitting period (47/110 participants, 42\%) and 1-month treatment (14/61 participants, 23\%) included pelvic cramping, urinary symptoms (including incontinence, urgency, frequency, and occasional difficulty in voiding), pelvic pain, vaginal spotting (fitting period only), and vaginal erythema, petechiae, or abrasions. It is noteworthy that adverse events associated with the VBC system decreased with time: $72 \%$ occurred during the fitting period with fewer reports at 1 month (20\%) and 3 months ( $8 \%$ ). No device-related adverse events were considered serious.

Furthermore, following 1-month use of the VBC system, participants reported significant improvement in Fecal Incontinence Quality of Life scores pertaining to lifestyle, coping behavior, and embarrassment, and in all categories of the Modified Manchester Health Questionnaire, except for the sleep and energy subscales where improvement was not significant. Most participants (90\%) expressed high satisfaction with their experience using the device with 54 of 55 women reporting they would recommend the VBC system to a friend.

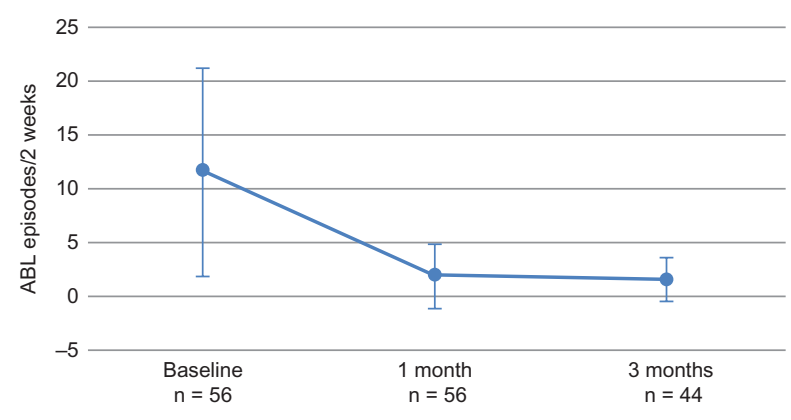

Figure 6 Within-subject efficacy of vaginal bowel control system wear on accidental bowel leakage $(A B L)$ episodes.

Notes: Data points represent mean \pm standard deviation. Data from Richter et al. ${ }^{23}$
Although this intervention study was short term, the results demonstrate the value of utilizing the vaginal space for rapid and effective management of ABL early in the treatment process.

\section{The LIFE clinical trial: impact of VBC system on bowel function parameters associated with $A B L$}

Because several aspects of bowel function influence QOL, the authors conducted a secondary analysis on the LIFE clinical trial data to evaluate the VBC system's effect on bowel movement frequency, urgency, stool consistency, and evacuation completeness. ${ }^{24}$ According to bowel diary entries at baseline and following treatment, 1-month use of the VBC system was associated with a significant decrease in bowel movements in those women reporting high frequency of bowel movements ( $>2$ per day) at baseline. In addition, fewer women reported liquid stools, urgency with bowel movements, and impaired evacuation. These data are particularly encouraging as these condition-specific symptoms of $\mathrm{ABL}$ are related to a woman's QOL and her ability to fulfill desired daily activities..$^{25,26}$

\section{The LIFE clinical trial: successful fitting characteristics of the VBC system}

The primary and secondary analyses of the LIFE clinical trial demonstrated the efficacy of the Eclipse System in treating incontinent episodes and bowel functions associated with ABL. However, the participants in the study represented only $55 \%(61 / 110)$ of the eligible enrollees, with the remaining potential subjects $(n=49)$ excluded primarily due to unsuccessful fitting of the device. Additional analysis of this excluded population was performed to aid in future patient selection and clinical protocol. ${ }^{27}$ Sixteen women were unsuccessful at the initial fitting and the remaining women $(n=33)$ complained of discomfort or displacement during the trial period or withdrew $(n=4)$. There were no differences in demographics, ethnicity, parity, menopausal status, body mass index, status of sexual activity, pelvic conditions, or comorbidity with irritable bowel syndrome between successful and failed fitting groups. Multivariate analysis indicated that women with previous pelvic prolapse surgery and women with shorter vaginal length were associated with an increased risk of an unsuccessful VBC insert fitting. These findings can be used to counsel patient expectations regarding the fitting experience. Furthermore, this information may guide consideration of new device size offerings to fit a higher percentage of patients in the future. 
Table I Overview of a clinical evaluation of a VBC system for the treatment of fecal incontinence in women (LIFE Study, NCT0 I655498)

\begin{tabular}{ll}
\hline Study type & Multicenter, prospective, open-label \\
\hline Device & A vaginal bowel control (VBC) system that consists of a vaginal insert and user-controlled, pressure-regulated pump. The vaginal \\
insert consists of a silicone-coated stainless steel base and posterior-directed balloon that provides reversible occlusion of the rectum \\
as controlled by the patient \\
Women between the ages of 19 and 75 years with: \\
Eligibility \\
A history of fecal incontinence for at least 6 months \\
A minimum of four incontinence episodes during the 2-week baseline period (assessed by patient diary) \\
insertion and removal of the vaginal insert, to participate in the study \\
Enrolled \\
Sixty-one women achieved successful fitting and entered treatment \\
One month of VBC system use and documentation of ABL symptoms in treatment bowel diary during the final 2 weeks of the wear \\
period \\
Optional 2-month extension of VBC system use with additional bowel diary entry during the final 2 weeks of wear (n=44 subjects) \\
Key findings \\
Eighty-six percent of per protocol participants met treatment success as reported by $\geq 50 \%$ improvement in ABL episodes \\
No serious adverse events during treatment. Reported adverse events were mild to moderate and resolved quickly \\
Participants reported higher quality-of-life ratings following VBC treatment \\
Women with ABL reported fewer liquid stools, less urgency with bowel movements, and improved evacuation \\
Women with high frequency of bowel movements at baseline ( $>2$ per day) noted a significant decrease in bowel movements following \\
I-month VBC treatment \\
Women with previous prolapse surgery and shorter vaginal length had greater difficulty in finding successful VBC fit
\end{tabular}

Abbreviation: $A B L$, accidental bowel leakage.

\section{Next steps for the Eclipse System - the LIBERATE clinical study}

The LIFE clinical trial provided initial demonstration of safety and efficacy of the Eclipse System following shortterm use in women with ABL (Table 1). Future directions include continued clinical investigations of a larger cohort of responders to the Eclipse therapy, with a longer duration of wear to evaluate the durability of its long-term safety and effectiveness. A multicenter, prospective, open-label, 1-year outcome clinical study is currently underway (ClinicalTrials. gov https://clinicaltrials.gov/ct2/show/NCT02428595, ID NCT02428595, LIBERATE Trial) ${ }^{28}$ to extend the support for the VBC system as a low-risk, patient-regulated treatment for ABL, restoring a woman's ability to control her bowel function and return to her daily activities.

\section{Conclusion}

$\mathrm{ABL}$ is a devastating condition for women with few effective options. A novel concept, the VBC system, offers a noninvasive option for women suffering from loss of bowel control. Multiple feasibility studies and a pivotal study have demonstrated the potential for a VBC system to offer immediate effectiveness with minimal risk and a positive impact on QOL. Further, the VBC has demonstrated a positive impact on symptoms associated with $\mathrm{ABL}$, including the urge to rush to a restroom for a bowel movement, loose stool, and frequent bowel movements.

\section{Acknowledgment}

This research was funded by the Wallace H. Coulter Translational Research Grant.

\section{Disclosure}

Since completion of this research, Dr Sokol has developed a consulting relationship with Pelvalon, Inc. The author reports no other conflicts of interest in this work.

\section{References}

1. Brown HW, Wexner SD, Segall MM, Brezoczky KL, Lukacz ES. Accidental bowel leakage in the mature women's health study: prevalence and predictors. Int J Clin Pract. 2012;66(11):1101-1108.

2. Bharucha AE, Dunivan G, Goode PS, et al. Epidemiology, pathophysiology, and classification of fecal incontinence: state of the science summary for the National Institute of Diabetes and Digestive and Kidney Diseases (NIDDK) workshop. Am J Gastroenterol. 2015;110:127-136.

3. Ditah I, Devaki P, Luma HN, et al. Prevalence, trends, and risk factors for fecal incontinence in United States adults, 2005-2010. Clin Gastroenterol Hepatol. 2014;12(4):636-643.e1-2.

4. Wu JM, Vaughan CP, Goode PS, et al. Prevalence and trends of symptomatic pelvic floor disorders in U.S. women. Obstet Gynecol. 2014;123:141-148.

5. Whitehead WE, Borrud L, Goode PS, et al. Fecal incontinence in US adults: epidemiology and risk factors. Gastroenterology. 2009;137: 512-517, 517.e1-2.

6. Meyer I, Richter HE. Impact of fecal incontinence and its treatment on quality of life in women. Womens Health (Lond Engl). 2015;11(2):225-238.

7. Wang A, Guess M, Connell K, Powers K, Lazarou G, Mikhail M. Fecal incontinence: a review of prevalence and obstetric risk factors. Int Urogynecol J Pelvic Floor Dysfunct. 2006;17(3):253-260.

8. Yu SW, Rao SS. Anorectal physiology and pathophysiology in the elderly. Clin Geriatr Med. 2014;30(1):95-106. 
9. Brown HW, Wexner SD, Segall MM, Brezoczky KL, Lukacz ES. Quality of life impact in women with accidental bowel leakage. Int J Clin Pract. 2012;66(11):1109-1116.

10. Norton C, Thomas L, Hill J, Group GD. Management of faecal incontinence in adults: summary of NICE guidance. BMJ. 2007;334(7608):1370-1371.

11. Brown HW, Wexner SD, Lukacz ES. Factors associated with care seeking among women with accidental bowel leakage. Female Pelvic Med Reconstr Surg. 2013;19(2):66-71.

12. Heymen S, Scarlett Y, Jones K, Ringel Y, Drossman D, Whitehead WE. Randomized controlled trial shows biofeedback to be superior to pelvic floor exercises for fecal incontinence. Dis Colon Rectum. 2009;52:1730-1737.

13. Norton C. Behavioral management of fecal incontinence in adults. Gastroenterology. 2004;126:S64-S70.

14. Brown SR, Wadhawan H, Nelson RL. Surgery for faecal incontinence in adults. Cochrane Database Syst Rev. 2013;7:CD001757.

15. Glasgow SC, Lowry AC. Long-term outcomes of anal sphincter repair for fecal incontinence: a systematic review. Dis Colon Rectum. 2012;55:482-490.

16. Wexner SD, Coller JA, Devroede G, et al. Sacral nerve stimulation for fecal incontinence: results of a 120-patient prospective multicenter study. Ann Surg. 2010;251(3):441-449.

17. Melenhorst J, Koch SM, Uludag O, van Gemert WG, Baeten CG. Sacral neuromodulation in patients with faecal incontinence: results of the first 100 permanent implantations. Colorectal Dis. 2007;9:725-730.

18. Hetzer FH, Bieler A, Hahnloser D, Lohlein F, Clavien PA, Demartines N. Outcome and cost analysis of sacral nerve stimulation for faecal incontinence. Br J Surg. 2006;93(11):1411-1417.

19. Bravo Gutierrez A, Madoff RD, Lowry AC, Parker SC, Buie WD, Baxter NN. Long-term results of anterior sphincteroplasty. Dis Colon Rectum. 2004;47(5):727-731; discussion 731-722.
20. Whitehead WE, Rao SS, Lowry A, et al. Treatment of fecal incontinence: state of the science summary for the National Institute of Diabetes and Digestive and Kidney Diseases workshop. Am J Gastroenterol. 2015;110:138-146.

21. Sokol ER. First-in-human acute assessment of a novel vaginal bowel control system for the treatment of fecal incontinence in women. $J$ Minim Invasive Gynecol. 2015;22(3):S53.

22. Pelvalon. A clinical evaluation of a vaginal bowel control system for the treatment of fecal incontinence in Women (LIFE). Available from: https:/clinicaltrials.gov/ct2/show/NCT01655498. Accessed April 26, 2016.

23. Richter HE, Matthews CA, Muir T, et al. A vaginal bowel-control system for the treatment of fecal incontinence. Obstet Gynecol. 2015;125:540-547.

24. Varma MG, Matthews CA, Muir T, et al. Impact of a novel vaginal bowel control system on bowel function. Dis Colon Rectum. 2016;59(2):127-131.

25. Markland AD, Greer WJ, Vogt A, et al. Factors impacting quality of life in women with fecal incontinence. Dis Colon Rectum. 2010;53:1148-1154.

26. Stenzelius K, Westergren A, Hallberg IR. Bowel function among people $75+$ reporting faecal incontinence in relation to help seeking, dependency and quality of life. J Clin Nurs. 2007;16(3): 458-468.

27. Matthews CA, Varma M, Takase-Sanchez M, et al. Clinical characteristics associated with successful use of a novel vaginal bowel control system for the treatment of fecal incontinence. Female Pelvic Med Reconstr Surg. 2016 (accepted and in press).

28. Pelvalon. A clinical evaluation of the Eclipse ${ }^{\mathrm{TM}}$ system, a Vaginal Bowel Control (VBC) therapy for fecal incontinence in women (LIBERATE). Available from: https://clinicaltrials.gov/ct2/show/NCT02428595 Accessed April 26, 2016.
Medical Devices: Evidence and Research

\section{Publish your work in this journal}

Medical Devices: Evidence and Research is an international, peerreviewed, open access journal that focuses on the evidence, technology, research, and expert opinion supporting the use and application of medical devices in the diagnosis, monitoring, treatment and management of clinical conditions and physiological processes. The identification of novel

\section{Dovepress}

devices and optimal use of existing devices which will lead to improved clinical outcomes and more effective patient management and safety is a key feature. The manuscript management system is completely online and includes a quick and fair peer-review system. Visit http://www. dovepress.com/testimonials.php to read real quotes from authors. 